\title{
Distributed Intelligent Lighting System by Performing New Model for Illuminance and Color Temperature in the Workplace
}

\author{
Mohammed Hajjaj, Mitsunori Miki \\ Graduate School of Science and Engineering, Doshisha University, Kyoto, Japan \\ Email:mhajjaj@mikilab.doshisha.ac.jp,mmiki@mail.doshisha.ac.jp
}

How to cite this paper: Hajjaj, M. and Miki, M. (2019) Distributed Intelligent Lighting System by Performing New Model for Illuminance and Color Temperature in the Workplace. Intelligent Control and Automation, 10, 1-12. https://doi.org/10.4236/ica.2019.101001

Received: November 14, 2018

Accepted: January 12, 2019

Published: January 15, 2019

Copyright $\odot 2019$ by author(s) and Scientific Research Publishing Inc. This work is licensed under the Creative Commons Attribution International License (CC BY 4.0).

http://creativecommons.org/licenses/by/4.0/

\section{(c) () Open Access}

\begin{abstract}
A new approach has been proposed to improve the performance of the intelligent lighting system by estimating personal illuminance and desired color temperature at the workplace. We are considering the problem of using the sensing devices manually for the intelligent lighting system. The lighting control system has not become useful without sensing devices to measure the provided illuminance and color temperature. In this paper, we have used the property of light for the color temperature to estimate the level of color temperature for each user at the workplace. The new method will give personal illuminance for each user at the workplace and decrease the power consumption of the environment as well. As a result, the proposed method of the intelligent lighting system has realized the target of illuminance and color temperature for each user at the workplace by adapting dimming levels using illuminance sensing information for each user. Thus, the energy of the workplace has reduced by using a distributed luminance to realize the target for each user.
\end{abstract}

\section{Keywords}

Intelligent Lighting System, Illuminance, Correlated Color Temperature, Chroma Meter, Automated Optimization Method

\section{Introduction}

Intelligent lighting systems have been applied in the daily life of firms and workplaces [1]. It has become more prevalent to provide appropriate lighting system while minimizing the energy of the environment. The lighting system does not only support the visual perception of users, but it also has an impact on the emotional and psychic biological in particular at the workplace [2]. Fur- 
thermore, the emerging technology of lighting systems has a dramatic impact of global warming by using the automated lighting control system for the environment [3].

Nowadays, a lot of different efforts in the lighting control system are becoming more prominent feature for the workplace to emphasize the productivity of each worker [2] [4]. The automated lighting control and intelligent lighting system has been used based on the principles of ergonomics. It has a positive influence on the performance, personal good health being, and intellectual productivity as well [4].

The automated lighting control system will enable users to change the level of luminance by using the ceiling lighting fixtures, a controller, and sensing devices. Hence, the intelligent lighting system has been used by applying a parallel distributed optimization method to change the level of luminance [5].

In this paper, we consider the distributed intelligent lighting system that relies on the automation control system to provide personal illuminance and correlated color temperature for each user in the workplace. Whatever each worker uses the sensing device to change the level of both illuminance and color temperature manually in the conventional method of the intelligent lighting system, so that the lighting control system cannot realize the desire of the user to provide an adequate level of luminance in the workplace [4] [6].

However, the intelligent lighting system becomes more practical and useful in providing the level of illuminance and color temperature for each worker respectively. This system has appeared to be much more effective in using a sensing device like a chroma meter sensing device. However, the intelligent lighting system using chroma meter sensing device is expensive. It has an obstacle to use the property of lights for color temperature and introduce the system at the workplace so that the intelligent lighting system has not become useful for workers.

Whatever the main problem of the intelligent lighting system is the inefficiency of achieving the desired luminance for each user. The importance of this paper has become in using a parallel distributed optimization method for the intelligent lighting system in the workplace. The need for adequate processing is to estimate the illuminance and color temperature while minimizing the power consumption of the workplace. Therefore, the using of an automated intelligent lighting system can help to provide the appropriate energy of each light which is required to achieve the target of illuminance and color temperature for each user.

We propose a new model of the intelligent lighting system to provide favorable luminance for each user in the office without using any sensing device. Thus, the performance of the new model for automating intelligent lighting system has been considered to provide personal illuminance and color temperature for each user in the workplace environment. This paper has the intent also to estimate illuminance and color temperature from the luminance information and realize 
the target for each worker by adapting dimming levels using illuminance sensor information while reducing the amount of the power consumption of workplace.

The rest of this paper proceeds as follows: in the first part, we discuss the structure of the lighting control system and the components of the intelligent lighting system as well. The second part of the paper is about the automated lighting control system and the formulation of the automation process following with proposed model for the system. Finally, verification and performed experiments have been conducted for the new model of the intelligent lighting system following with the results in details.

\section{Related Works}

A lot of experiments have been conducted for the intelligent lighting systems, and also many works have done to increase the efficiency of users in the workplace since the automated lighting system is of interest to the office environments.

The contribution of work in [7] has considered the problem of controlling the multiple luminaries with collocated occupancy and light sensors. This is was by attaining illumination levels higher than specified values while minimizing power consumption using different types of least squares to estimate illuminance at the workplace. Besides the works in [6] [8], another optimization method based on simulated annealing has been used to change the luminous intensity of light. However, the power consumption by using the method of the work [8] is reduced while satisfying the target illuminance and target color temperature.

Therefore, it is common practice in the lighting control system to provide the level of illuminance and color temperature based on the health condition of the user and choosing the time for the system overall [9] [10]. As well as in contribution [11], another approach has been used to measure the color temperature for multiple lights cases. This approach was by using a new model of the automated lighting control system, so the work has found that there is related to the corresponding weight of occupied area by the user for the desks.

The contributions of papers have been achieved to increase the performance of the lighting control system, and also to carry out the optimal lighting output for users in workplace environments. However although, this paper is contributing to estimate the level of color temperature of light for each user automatically and individually without using sensing devices to measure the level of color temperature, as well as to provide personal illuminance and desired color temperature while minimizing the energy in the workplace.

\section{Intelligent Lighting Model}

In this section, we review the system architecture of the intelligent lighting system; and the formalizing of the optimization method; and the automation system as well. Furthermore, we review the proposed model of the intelligent lighting system exists on the illuminance and the color temperature simultaneously. 


\subsection{Model Architecture}

The intelligent lighting system has the components as depicted in Figure 1: 1) lighting setting and ceiling lighting fixtures; 2) lighting apparatuses module or power meter; 3) sensing device module as chroma meter; 4) controller module. The lighting fixture is installed to provide and reach proper light levels and uniform light distribution for each user in the workplace.

The light control system can change the lighting pattern for the desks based on the values of illuminance and color temperature data which are received by the chroma meter sensing device. Each desk including one sensing device is located in the workplace and influenced by lights so that the illuminance value describes the quantity of luminous flux to one square meter of the desk.

Therefore, the values of illuminance have affected based on the distance between the emitted light and the located sensing device in the desk. Hence, the influence coefficient is the main effective variable to measure the power of light to the sensing device. Then, each user can get the level of adequate luminance based on the physical locating of the desk. However, the physical locating of the desk has an effect to get the target as desired, too [12].

The lighting fixture in the workplace has installed with two different light sources of color emitted from the lamp appears as a natural cool, or a warm light source. Then the worker can change the color appearance in the workplace as the desired. Furthermore, the most common use of color temperature for the office is in the range 2000 to 6000 Kelvin degree. In this paper, the color light sources are available in the range from 3025 to 4850 Kelvin for warm to cool color light source. The color of cool or white light is the most use of the light source of color, and it has become the most popular use in the environments of the workplace.

In the conventional model of the intelligent lighting system, each user can change the desired value of illuminance and color temperature by using the sensing device in the desk manually.

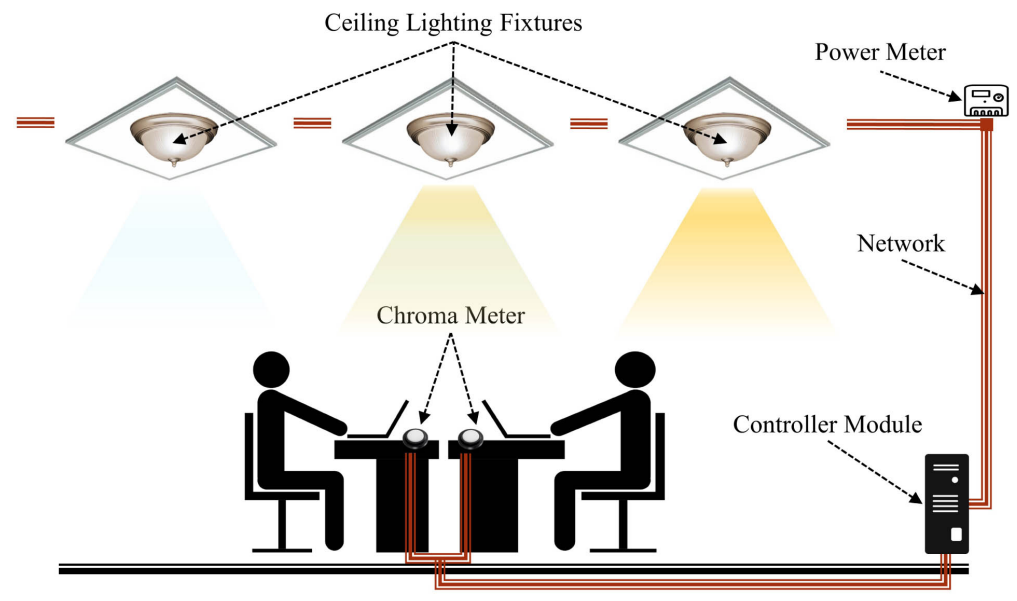

Figure 1. The architecture model of the lighting control system with two color light sources and collected chroma meter sensing devices in each desk. 


\subsection{The Formulation of Lighting Control System}

At first, the lighting control system has used the illuminance and the color temperature as a part of the formulation. The first part, the system measures the illuminance by using the ratio of luminous power which falling on the surface of the desk in lux (lx) as in (1):

$$
I=\sum_{i=1}^{n} R_{i j} L_{i}
$$

where $I$ is the value of illuminance measured by one illuminance sensing device; $i$ refers to the light; $j$ refers to the sensing device; the $R_{i j}$ is the influence coefficient between the light and sensing device; $L_{i}$ refers to the power of luminance for the light.

The second part of the lighting control system is the correlated color temperature. The system calculates the correlated color temperature by using the associated function for both colors light sources provided in lamps. In the lamps, there are two light sources of color, and both of them has an associated linear relationship so that the ratio of illuminance for one color light source to the total illuminance can depict the value of correlated color temperature in Kelvin (K) as in Figure 2.

\subsection{The Automation and Lighting Control System}

The objective function in the automated lighting control system for each light $f_{i}$ is derived based on the values of the power consumption; the illuminance constraint; and the correlated color temperature, as depicted in the model (2).

$$
f_{i}=p+w_{g} \times \sum_{i=1}^{n} g_{i j}+w_{h} \times \sum_{i=1}^{n} h_{i j}
$$

where $p$ is the power consumption; $g_{i j}$ is the illuminance constraint; $h_{i j}$ is the color temperature constraint. The value of weighting factor $w$ is used to make equilibrium between each condition. The $i$ refers to the light; $j$ refers to the sensing device.

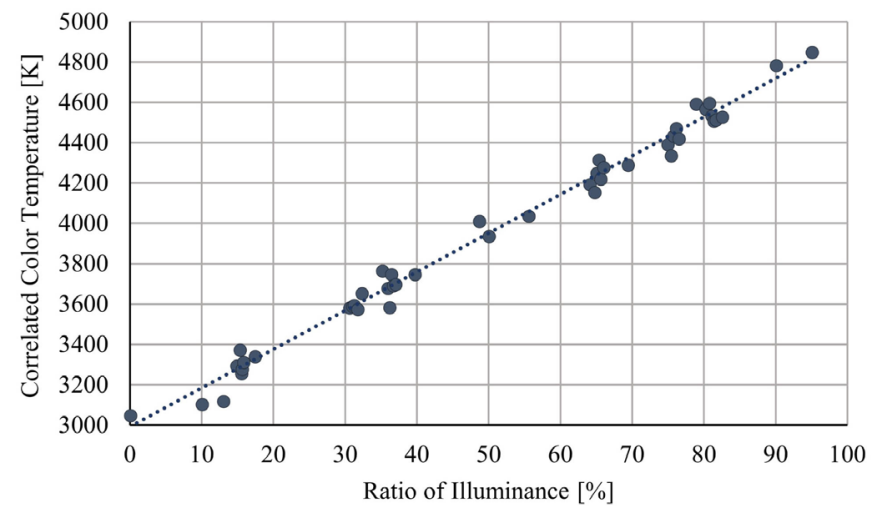

Figure 2. The correlated color temperature of cool color source light calculated by the ratio of the illuminance for cool color source light to the total illuminance of the cool and warm source light. 
The illuminance constraint and the correlated color temperature constraint have affected based on the influence coefficient and the target for each illuminance and color temperature, as in (3), and (4).

$$
\begin{aligned}
& g_{i j}=R_{i j} \times\left(I c_{j}-I t_{j}\right)^{2} \\
& h_{i j}=R_{i j} \times\left(C c_{j}-C t_{j}\right)^{2}
\end{aligned}
$$

where the $R_{i j}$ refers to the influence coefficient; the $I c_{j}$ and $I t_{j}$ refer to the current and target illuminance; and the $C c_{j}$ and $C t_{j}$ refer to the current and target color temperature.

The values of the influence coefficient are classified to categories based on the size and capacity of the workplace. In general, the lights are affected by three classes based on the values of the influence coefficient: high, medium, and low effectiveness. The three classes have recognized the effect on the eyes of the user. Whenever the sensing device is close to the light, the influence coefficient will be high, other the value will be medium or low based on the distance between the sensing device and the light.

\subsection{Probable Solutions of the Automated System}

The intelligent lighting system is a part of the automated lighting control. Then the automated lighting system can obtain the target value of illuminance and color temperature using a small amount of luminance to obtain the target for each user in the office.

However, the intelligent lighting system has used the method of Adaptive Neighborhood Algorithm using Regression Coefficient (ANA/RC) to realize the luminance intensity in respect to the target of each user. The algorithm can enable the intelligent lighting system to get the target in a short time by changing the rate of luminance in the dimming process for each light. This process could help to decrease the power consumption of the environment.

The distribute luminance has been given by the following approach: at first, initialize all luminance in a minimum value. Then the current illuminance and color temperature values for each sensing device are figured. However, the constraints for each sensor are determined by using the target and current values of illuminance and color temperature. After that to get the best solution for each light, the lighting control system has changed the current values of luminance to realize the target. Therefore, ANA/RC is applied during the process to realize the target of the intelligent lighting system.

In the dimming process for the luminance intensity, the distribution of probable solutions of changing the luminance in the lights depends on the value of luminance intensity. It has been classified into three categories to realize the target of the system, as shown in Figure 3. The luminance intensity increases or decreases rapidly depending on the priority. For example, if the current luminance is low and the required target is greatly different, the lighting control system will increase the luminance intensity rapidly, otherwise, the system will decrease it 


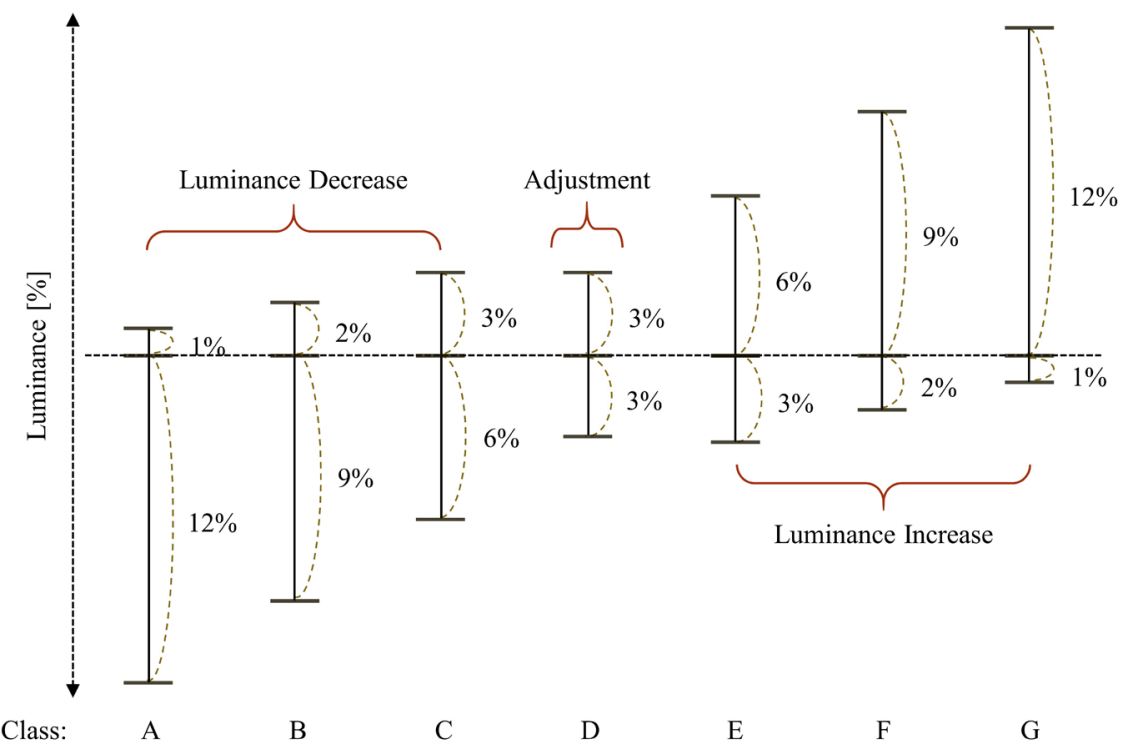

Figure 3. The variation of probable solutions of changing luminance categorized in classes to luminance decrease, luminance adjustment, and luminance increase.

rapidly. Furthermore, the system will use the average illuminance for the luminance intensity is not in high or low level.

Therefore, the rules are designed to find the variety of probable solutions as depicted in Table 1 . The decreasing luminance has categorized into three categories: rapid luminance decrease (Class A); medium pace luminance decrease (Class B); and slow luminance decrease (Class C). In another side, the increasing luminance has also categorized into three cases: rapid luminance increase (Class $\mathrm{G}$ ); medium pace luminance increase (Class F); and slow luminance increase (Class E). Otherwise, luminance is in average for all different cases, called luminance adjustment (Class D).

\section{Verification Experiments}

The intelligent lighting system has conducted in the experimental environment as in Figure 4. The room of experiment has twelve lamps for luminance, and six desks located randomly for workers. Each desk has one sensing device to measure the values of luminance. The lamps have installed in the ceiling fixture with two light sources of color for each lamp: the cool color and warm color light source. All lamps can provide luminance in range $1350 \mathrm{~cd}$ for cool luminance level, and $1190 \mathrm{~cd}$ for warm luminance level. Besides, the lamps can give the color temperature in the range $3025 \mathrm{~K}$ for a warm color to $4850 \mathrm{~K}$ for a cool color light source.

However, the experiments have been conducted in a normal situation to verify the efficiency of the intelligent lighting system. We set the target of illuminance and color temperature for all sensing devices, then the constraints of each illuminance and color temperature have been figured by the intelligent lighting system using the proposed method. The target values of illuminance for all 


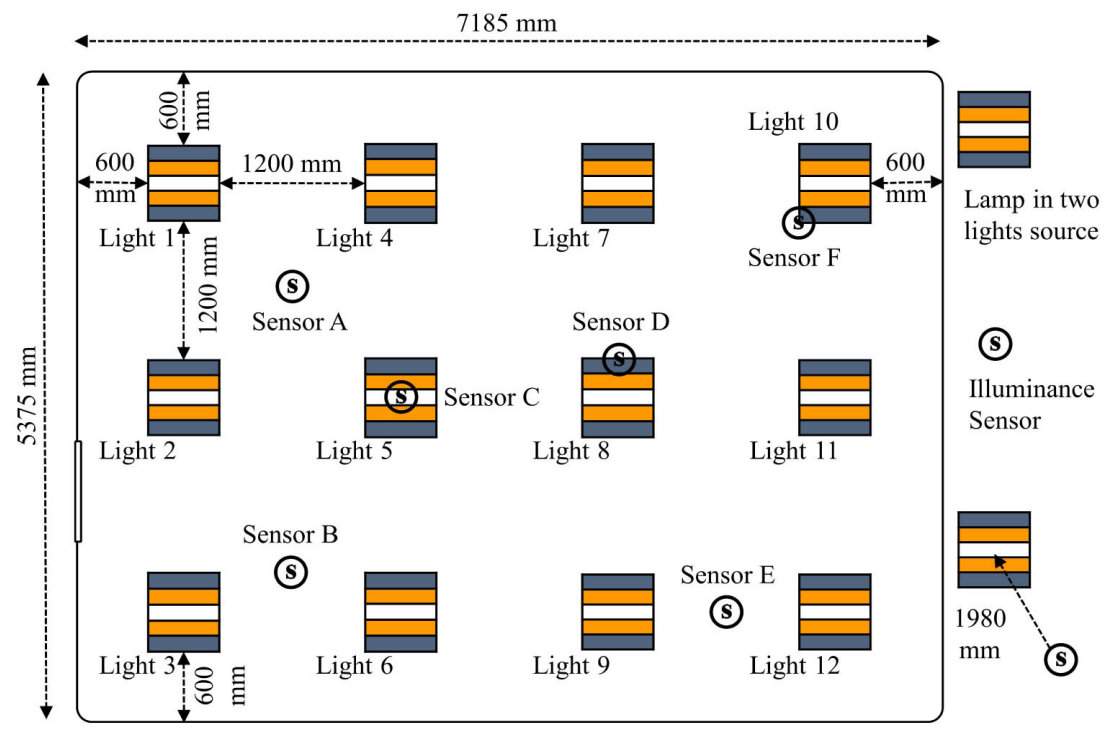

Figure 4. The experimental environment of the lighting control system model with twelve lamps in two color source lights, and six distributed sensing devices.

Table 1. The classification design and rules for distributing luminance while the dimming process of lights based on the illuminance constraints and influence coefficient.

\begin{tabular}{lcccc}
\hline \multirow{2}{*}{ Rules (Classes) } & & \multicolumn{3}{c}{ Illuminance Constraint } \\
\cline { 3 - 5 } & & High & Medium & Low \\
\hline \multirow{3}{*}{ Influence Coefficient } & High & $\mathrm{D}$ & $\mathrm{D}$ & $\mathrm{E}$ \\
& Medium & $\mathrm{C}$ & $\mathrm{C}$ & $\mathrm{D}$ \\
& Low & $\mathrm{C}$ & $\mathrm{B}$ & $\mathrm{A}$ \\
\hline
\end{tabular}

sensing devices have been set in the values: $300 \mathrm{~lx}, 500 \mathrm{~lx}$, and $700 \mathrm{~lx}$, and the target values for color temperature have been set to the values: $3500 \mathrm{~K}, 4000 \mathrm{~K}$, and $4500 \mathrm{~K}$. After using the new method of the intelligent lighting system, we have obtained the current values of illuminance and color temperature as shown in Figure 5.

The target of illuminance and color temperature based on the history have been got in 200 seconds approximately. Occasionally, the system can make the target after 200 seconds based on the physical locations of sensing devices which used to conduct the experiments, and the capacity size of the experimental environment. Moreover, the difference between the current and target values for illuminance and color temperature are not high. Whatever the average error of illuminance values for all sensing devices is in the range $30 \mathrm{~lx}$, and this value cannot be noticed by each user in the workplace. As well as, the average error of color temperature values are about $200 \mathrm{~K}$, and this value is too small which cannot be recognized by the human eyes by each user at the workplace, as in Table 2.

Therefore, performing model of the distributed intelligent lighting system using the new approach of the automated system can be applied in the real office and the large workplace environment. The system could attain the target of 

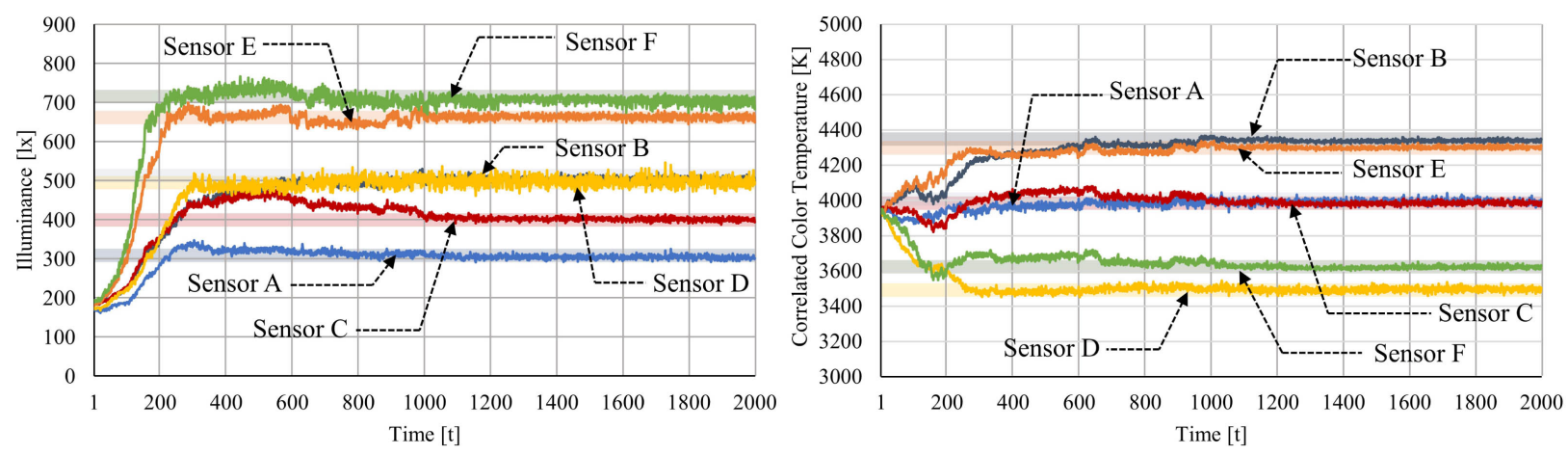

Figure 5. The history of the intelligent lighting system for six sensing devices using the proposed method for automated lighting control. The history of illuminance values (Left), and the history of correlated color temperature values (Right) in realizing the target values of the system.

Table 2. The history of illuminance and correlated color temperature for the six sensing devices in comparing with the target. Ic: current illuminance; It: target illuminance; CCTc: current correlated color temperature; CCTt: target correlated color temperature.

\begin{tabular}{ccccc}
\hline \multirow{2}{*}{ Sensing Devices } & \multicolumn{2}{c}{ Illuminance Constraint } & \multicolumn{2}{c}{ CCT Constraint } \\
\cline { 2 - 5 } & Ic $(\mathrm{lx})$ & It $(\mathrm{lx})$ & CCTc $(\mathrm{K})$ & CCTt $(\mathrm{K})$ \\
\hline Sensor A & 300.03 & 300 & 3979.06 & 4000 \\
Sensor B & 508.36 & 500 & 4336.96 & 4500 \\
Sensor C & 403.10 & 400 & 3983.79 & 4000 \\
Sensor D & 497.32 & 500 & 3493.98 & 3500 \\
Sensor E & 630.13 & 600 & 4297.63 & 4500 \\
Sensor F & 714.43 & 700 & 3616.16 & 3500 \\
\hline
\end{tabular}

illuminance and color temperature in advance for each sensing device. Thus, the system has met the target while minimizing the energy of the environment. In the real office, the experimental results are similar to the large office with more lights and increasing the number of users.

Therefore, the new model of the intelligent lighting system is very effective and efficient in the large offices as well. The system has attained the target of illuminance and color temperature in a short time for each user. Thus, the user can act in a few time to start the work in a good environment for each user to interact in the office.

In the large office, the converging time has not become so long to get the target, and it is similar to the result of the experimental office. The sensing device has affected with a few numbers of surrounding lights. Thus the intelligent lighting system has a divided action for a large number of lights and sensing devices in the office. The problem divided into a small problem in the intelligent lighting system for the large offices, but the problem cannot be divided into a small problem if the sensing device has affected by lights with a very long distance.

On another side, the luminance level for each light has distributed based on the required target of illuminance and color temperature, and the influence coefficient for each sensing device to the light as shown in Figure 6. Thus, the 


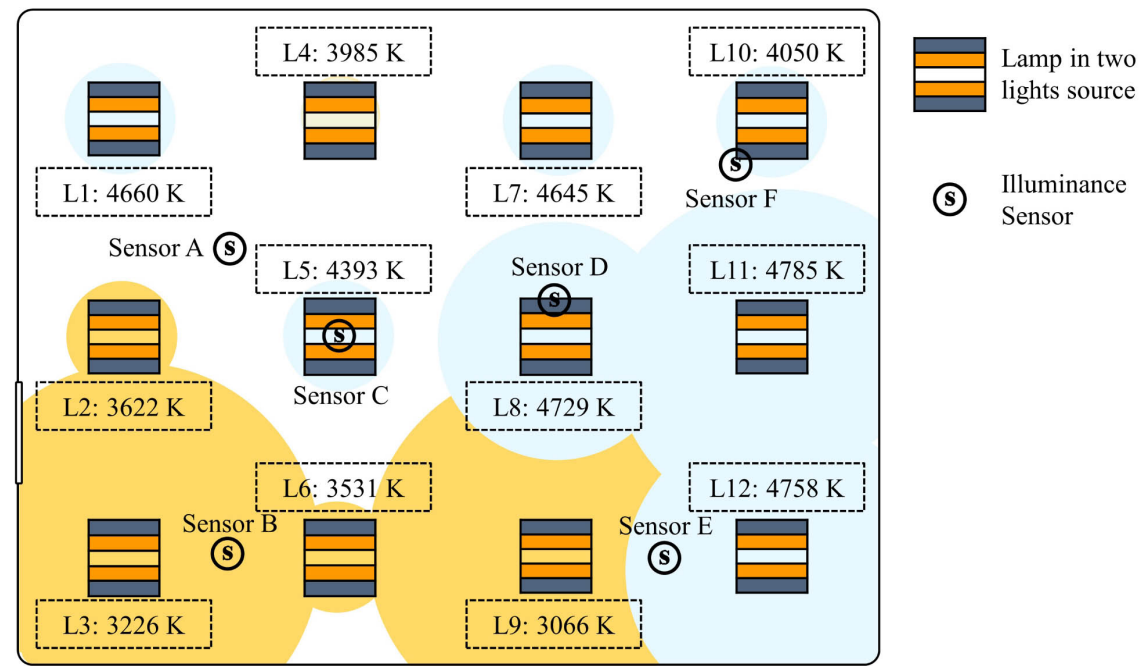

Figure 6. The distribution of luminance values for all lights, as well as the values of color temperature which could realize by each light individually.

intelligent lighting system has used the smallest required amount of energy to get the target of illuminance and color temperature for each user. Thence, all lamps which close to the sensing device are brightly turned on to meet the target of illuminance and color temperature.

Moreover, the system can find the best solution to reach the target of each user under any physical constraint. In an example, the target values of illuminance for two closed sensing device was in $300 \mathrm{~lx}$, and $700 \mathrm{~lx}$, the intelligent lighting system could give $350 \mathrm{~lx}$, and $630 \mathrm{~lx}$ for both sensing devices respectively. Then, the changed values between the target and current illuminance even in the values of color temperature are not too high. Thus, the provided values by the system are the best solution of illuminance for both closed sensing device, so that the system has obtained the best values of the target to keep the energy of the environment in the smallest value under the physical constraints. Therefore, the behavior of the intelligent lighting system has been studied by conducting performed experiments in different cases to measure the efficiency of the system under physical restrictions.

\section{Conclusions}

We consider the problem of the conventional intelligent lighting system using sensing devices. The sensing device is the main component to obtain the target of illuminance and color temperature for each user while minimizing the energy at the workplace in the intelligent lighting system. In this paper, the new model of the automated lighting system without using sensing devices has attained the target in advance for each user in the office. However, the performing model of the intelligent lighting system has provided personal illuminance and desired color temperature for each user in the workplace.

Therefore, the new model has used the best level of luminance to get the target in comparing with the conventional lighting system. The system also has carried 
out the goal of the environment while minimizing the energy, and it will help in global warming. Therefore, the new model of the system can estimate the color temperature without sensing devices by using the regular relationship between the ratio of natural cool and warm source light.

Thus, the distributed intelligent lighting system using the proposed method can be applied in the real office and large space of the workplace environment. Furthermore, the new model contributes to the office field for maintaining and enhancing productivity in the workplace. Then the use of intelligent lighting system helps users in doing the work more efficiently in good health away from the stress of lights in the workplace.

\section{Conflicts of Interest}

The authors declare no conflicts of interest regarding the publication of this paper.

\section{References}

[1] Castillo-Martinez, A., Medina-Merodio, J.-A., Gutierrez-Martinez, J.-M., Aguado-Delgado, J., de Pablos-Heredero, C. and Otón, S. (2018) Evaluation and Improvement of Lighting Efficiency in Working Spaces. Sustainability, 10, 1110. https://doi.org/10.3390/su10041110

[2] Cadena, R. (2006) Automated Lighting: The Art and Science of Moving Light in Theatre, Live Performance, Broadcast, and Entertainment. Taylor \& Francis, Thames.

[3] Mills, P.R., Tomkins, S.C. and Schlangen, L.J. (2007) The Effect of High Correlated Colour Temperature Office Lighting on Employee Wellbeing and Work Performance. Journal of Circadian Rhythms, 5, 2. https://doi.org/10.1186/1740-3391-5-2

[4] Noguchi, H. and Sakaguchi, T. (1999) Effect of Illuminance and Color Temperature on Lowering of Physiological Activity. Applied Human Science, 18, 117-123. https://doi.org/10.2114/jpa.18.117

[5] Pandharipande, A. and Newsham, G. (2018) Lighting Controls: Evolution and Revolution. Lighting Research \& Technology, 50, 115-128. https://doi.org/10.1177/1477153517731909

[6] Tanaka, S., Yoshikata, M., Miki, M. and Hiroyasu, T. (2009) An Evolutional Optimization Algorithm to Provide Individual Illuminance in Workplaces. 2009 IEEE International Conference on Systems, Man and Cybernetics, San Antonio, 11-14 October 2009, 941-947. https://doi.org/10.1109/ICSMC.2009.5346094

[7] Borile, S., Pandharipande, A., Caicedo, D., Schenato, L. and Cenedese, A. (2017) A Data-Driven Daylight Estimation Approach to Lighting Control. IEEE Access, 5, 21461-21471.

[8] Tomishima, C., Miki, M., Ashibe, M., Hiroyasu, T. and Yoshimi, M. (2010) Distributed Control of Illuminance and Color Temperature in Intelligent Lighting System. International Conference on Artificial Intelligence and Soft Computing, Springer, New York, 411-419. https://doi.org/10.1007/978-3-642-13232-2_50

[9] Taniguchi, Y., Miki, M., Hiroyasu, T. and Yoshimi, M. (2011) Preferred Illuminance and Color Temperature in Creative Works. 2011 IEEE International Conference on Systems, Man, and Cybernetics, Anchorage, 9-12 October 2011, 3255-3260. https://doi.org/10.1109/ICSMC.2011.6084171

[10] Miki, M., Motoya, Y., Ikegami, H. and Fujimoto, S. (2013) Combination of Pre- 
ferred Illuminance and Preferred Color Temperature for Work. 2013 IEEE International Conference on Systems, Man, and Cybernetics, Manchester, 13-16 October 2013, 4577-4582.

[11] Xinping, B., Guanglei, Z. and Jichuan, Z. (2014) Illuminance and Color Temperature Control in Intelligent Lighting System. Ricoh Software Research Center (Beijing) Co., Ltd., Beijing.

[12] Ikegami, H., Kuwajima, S., Miki, M. and Aida, H. (2014) An Extraction Method of Influential Lightings for Illuminance Sensors on an Intelligent Lighting System in Large Office. Proceedings on the International Conference on Artificial Intelligence (ICAI), Las Vegas, 21-24 July 2014, 1. 